\title{
Research of Ice-melting Monitoring System Based on Internet of Things and Image Processing Techniques
}

\author{
SUN Peng \\ School of Automation, Wuhan University of \\ Technology \\ WHUT \\ Wuhan, China \\ e-mail: 948783205@qq.com \\ ZHANG Li-xin \\ School of Automation, Wuhan University of \\ Technology \\ WHUT \\ Wuhan, China \\ e-mail: xfxzlx@qq.com
}

\author{
WANG Shuai \\ School of Automation, Wuhan University of \\ Technology \\ WHUT \\ Wuhan, China \\ e-mail:623924970@qq.com \\ LI Zhi-jun \\ School of Automation, Wuhan University of \\ Technology \\ WHUT \\ Wuhan, China \\ e-mail: jzhli@whut.edu.cn
}

\begin{abstract}
With the fast development of electricity system in China, the lack of a monitor method to ensure the stability and safety of electric transmission simultaneously comes with the strengthened construction of power supply. Besides, the Icing phenomenon is common to see in the electricity system and it brings enormous damages and negative social influence. However, the current Icing monitoring system needs substantive energy to consume and it is expensive to maintain. Accordingly, having analyzed the existing project, the author designed a new method which is based on the detecting pattern of Internet of things, including CCD image capture, assisting sensor detection, information collection via ZigBee Internet, DSP image processing and GPRS centralized transmission, to automatically process and analyze the icing situation through image processing technique.
\end{abstract}

Keywords- ice-melting; internet of thing; image processing techniques; monitoring

\section{INTRODUCTION}

The geographically situation in China is special for the impact from monsoon climate and the icing in high voltage transmission line is very widespread. It is well known that the icing in electric transmission line can easily give rise to the threat of the wave 、 breakage collapse 、 insulator arc-over 、 line lightning tripping, and even the outage of electric net which risks the safe operation of electric system and result huge economic loss[1-4]. Precisely, icing phenomenon in high voltage transmission line is found in places where is in rural area of awful weather, which makes it harder to detect icing the icing parameter. However, the main method today is focused on precaution. In China, we mainly build icing detecting station 、 icing weather model and icing mechanical model, the three measures, to enhance Improve the ice tower carrying capacity and tensile strength of the wire v rationalize the select of safety parameter of guide line and optimize the planned route by differentiated transforming the icing route[2]. Considering the mechanism of icing is complex and the diversity of icing weather models, there is a no unanimous description yet and most of the models are deduced on the long observation from a certain region which would weaken the universality of it.So there lacks a targeted plan aimed at the special wire of pylon. In 2008, the massive ice damage led to the massive icing, it is obviously patrolling and climbs the tower to check the wire by human lacks efficiency

Taking all the situation abovementioned into account, the author can still find the problems such as high cost、 narrowly detecting extent and outdated data in existing method after looking for some relative materials. Consequently, this paper is intended to propose a practical and optimized method to complete the detection on icing phenomenon in high voltage transmission line. 


\section{THE ANALYSIS ON ICING DISASTER}

\section{A. The fault analysis on disaster}

Take the condition of icing $20 \mathrm{~mm}, 426$ elevation, $5^{\circ} \mathrm{C}$ temperature and $10 \mathrm{~m} / \mathrm{s}$ wind speed in a certain line in Yunnan Province as an example. The Aluminum alloy wire of LGJ-150/20 as a simulation. (The physical character can be seen in figure1.) The represented gears $\mathrm{Ld}$ is $417 \mathrm{~m}$ and the output power is $35 \mathrm{kv}$.(The condition is annual average temperature and the icing b is $0 \mathrm{~mm}$, temperature is $15^{\circ} \mathrm{C}$, wind speed is $0 \mathrm{~m} / \mathrm{s}$, the represented gears Ld is $417 \mathrm{~m}$. According to the state equation of wire:

We can calculate the maximum horizontal force under the conditions is and the result is the $84 \%$ of the breakdown stress of the wire. If the influence of height difference is considered, the wire stress of suspension point is $240.95 \mathrm{Mpa}$, which is $89 \%$ that of the breakdown stress of the wire and it is far more excessive compared with the criteria that the maximum horizontal force is supposed to be less than the $60 \%$ of the breakdown stress under heavy ice area. However, when the ice falls from the icing wire, the suspension point would exert huge longitudinal impacts load and damage the wire to a great extent. Having suffered the breakdown stress close to the extreme, the wire would be severely damaged and the icing phenomenon comes.

\section{B. The device and the principle of identification module of picture processing}

Icing images can be captured by CCD camera and be sent to DSP through ZigBee with processed by decoding chip. Then, the ZigBee use the internal added 8051 and the parallel serial communication to transfer the images at the speed of more than $20 \mathrm{~kb}$ to meet the requirements.

DSP can roughly calculate the thickness of icing by serious steps. Firstly, making smoothing and marginal detection on the images captured. Then, recording down the coordinate of boundary contour points in images by reasonable binarization processing and boundary following. Compared with the change of coordinate of wire and insulator caused by icing, the thickness of icing can be calculated by the diameter of wire line calibration ultimately ${ }^{[3]}$.

In detail, the thickness can be calculated by firstly, accumulating the pixel of wire in up and down marginal icing layer. Then, calculating the specific value from the re-accumulating the pixel of the diameter of wire and the data in the first step. Finally, the relationship between diameter of wire and the thickness of icing can be found as well as the average value of icing thickness of the wire.

Where The average thickness was defined as $\mathrm{H}$.

\section{THE DESIGN AND RESEARCH OF SYSTEM PLAN}

The design drawing of the system is presented in one.

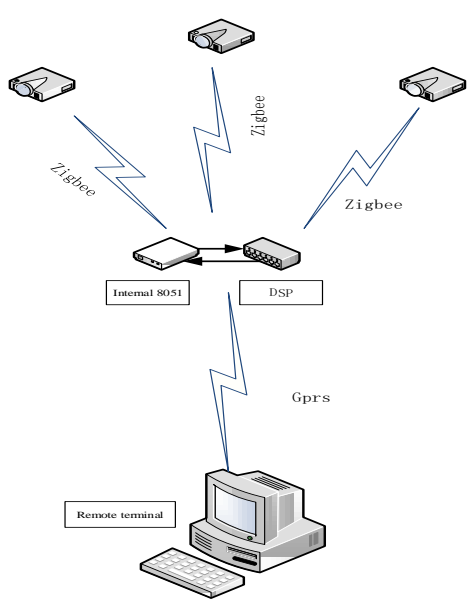

Figure 1 The design drawing of the system

Aimed at the abovementioned context and materials, the author has designed an optimized plan consists the electric transmission line to detect icing with taking the detective context, application extent, the construction cost and the technical condition into account. This plan is divided into three parts: the acquisition terminal of camera, information transport network, data processing module and the monitor and management centre. Information can be received and mutual communication can be achieved through getting single width images gathered by CCD, through decoding device to be transformed into corresponding format, through wireless transformation to nodes with DSP and ZigBee included after mark added by ZigBee, through the internal enhancement mode 0805 to make serial pot communication model. Meanwhile, the GPRS module carried within 0805 will send the information processed by DSP to the monitoring center to realize the supervisory control on icing route.

\section{A. Systematic hardware design plan}

1) The overall plan of hardware

This plan abandoned the existing method of processing data by connecting DSP directly via data line from single camera. Centralized collection and processing of data by ZigBee can substantially improve the efficiency, fulfill the goal of one set equipment management within a single net and greatly decrease the cost of construction and maintaining. The frame diagram of this hardware system is presented figure two. 


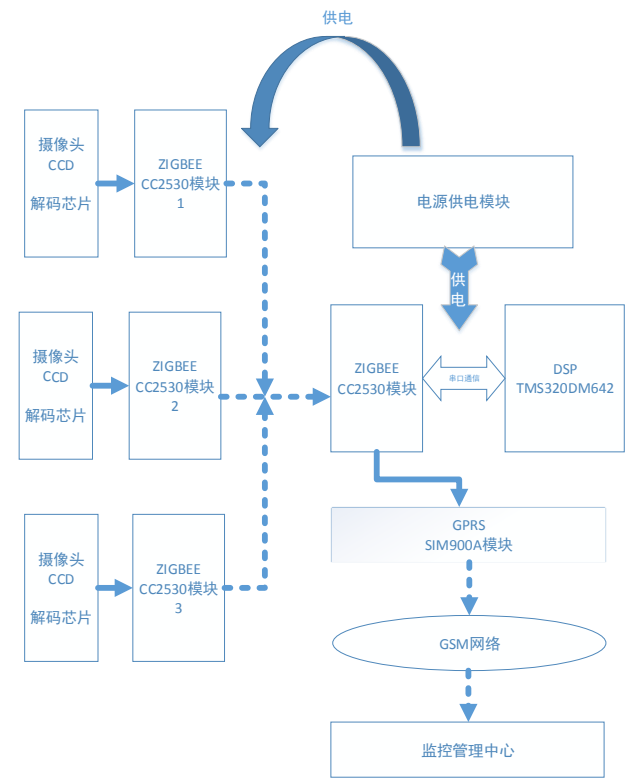

Figure 2 The frame of this hardware system

2) The acquisition terminal of camera

The decode module is TVP5150 and this module is a ultra-low power consumption NTSC/PAL/SECAM video decoder produced by TI. TVP515 module takes the standard interface of $2.54 \mathrm{~mm}$ space width and 30 pin ISI and leads to two ways: BNC and AV interface. This module will transform the data flow exporting from camera in PAL format to digital color difference signals and finally to DSP through the internal enhancement mode 8051 in ZigBee serial unvarnished transmission

3) The acquisition terminal of camera

This plan achieves the centralized processing of information by using various means of communication to make every way constitutes a net structure and substantially saves the cost, facilitates the management and reduces the difficulty of maintaining. The general block diagram of communication network is shown in figure three.

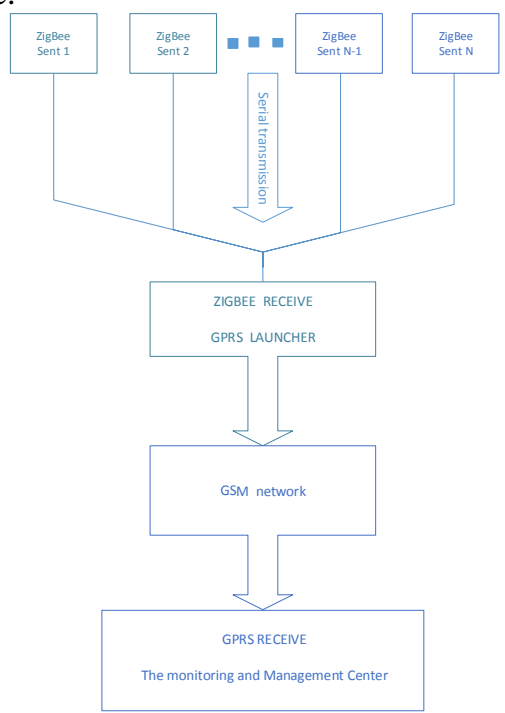

Figure 3 The general block diagram of communication network
The ZigBee takes the CC2530 from TI Company and obtains the flash memory of $256 \mathrm{~kb}$, the integration of 8KBRAM and the enhancement mode 8051 with eightcore MCU. According to the development board and test data provided by jiuzhou net limited company, when emission power is $2.5 \mathrm{mw}$ and the distance is $200 \mathrm{~m}$, the transmission speed is up to $256 \mathrm{~kb}$, the receiving electricity is less than 25milliapere and the emission current is less than 40milliapere. The character of lower power and large coverage successfully suits this plan.

4) Data processing module

Data processing module is made up of the internal enhancement mode 8051 and the TMS320DM642chip from TI Company. Through receiving information from ZigBee, transforming information via serial port to DSP and the EMIF port, the SDRAM and Flash storage are expanded. The external space can be expanded into four storages by EMIF port: CE0 - CE3. Also, the externally expanded storages can be mapped in different spaces. In detail, SDRAM is mapped into CEO space to store images and data. Flash is mapped in CE1 space to store systematic procedure.

When the system is operating, DSP will carry out application programs to return to 8051 and be sent to monitor centre in the form of data network by serial port of SIM900A under the guidance of bootloader through receiving different ZigBee information, compiling zone bit and orderly being transformed to DSP. Finally, the thickness of icing in every zone can be supervised.

\section{B. The design method of systematic software}

As a certain period of time is needed for freezing, the enhancement in quantity of marks in different spots benefits the calculation and costs. Besides, sequential processing of images is more scientific for estimating the thickness of icing. As showed in figure four, the author takes the systematic software chart.

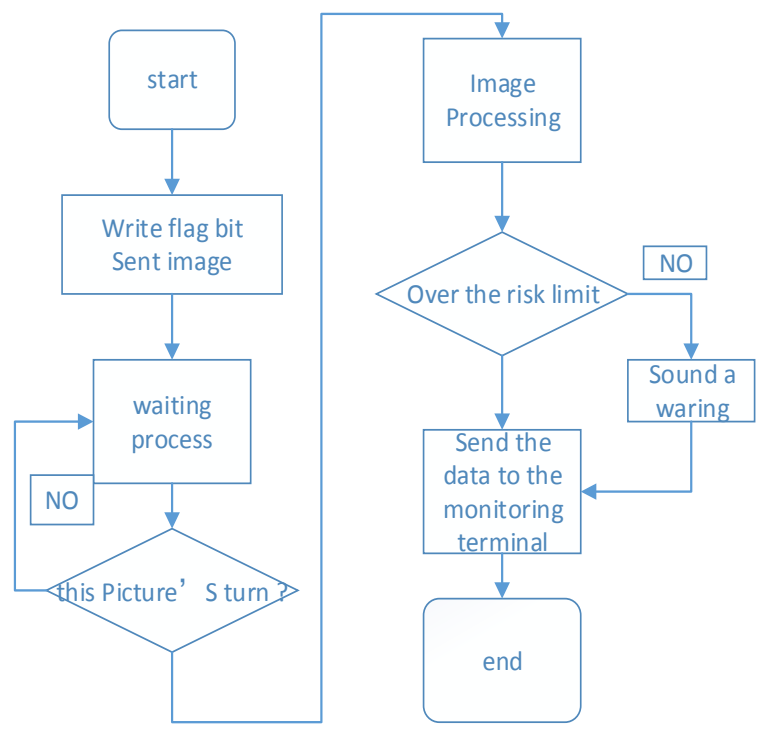

Figure 4 the systematic software chart

\section{1) ZigBee communication module}

ZigBee initializes the net through coordinator and follows several steps such as detecting coordinator, 
channel scanning, and allocation of network parameter, operation of new network and permission of join from device. After that, node is prepared to join network by relevance MAC layer and finally join the network with appointed father node. Flow diagrams are figure five and six.

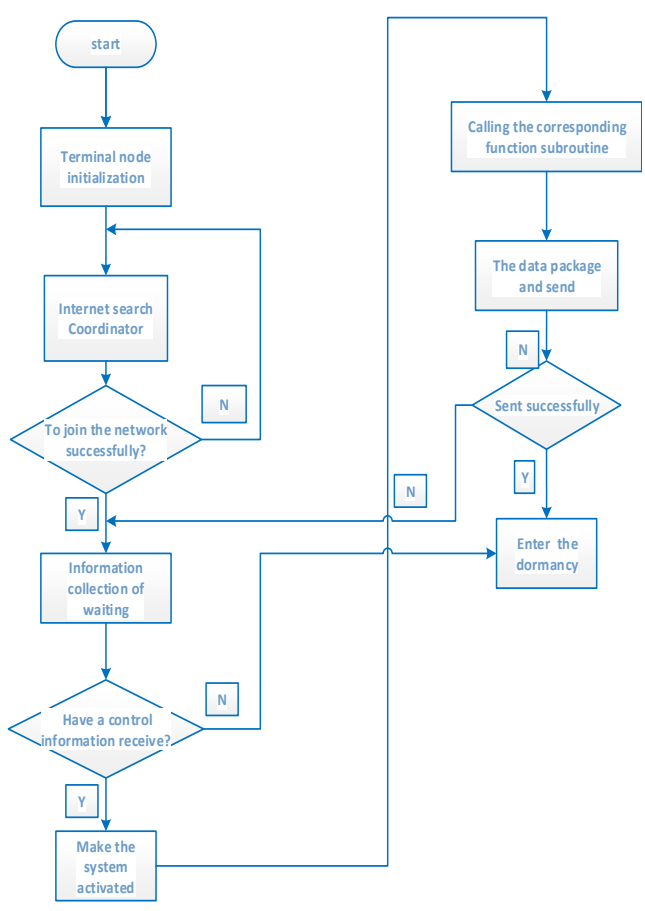

Figure 5 The ZigBee terminal node program flowchartZigBee

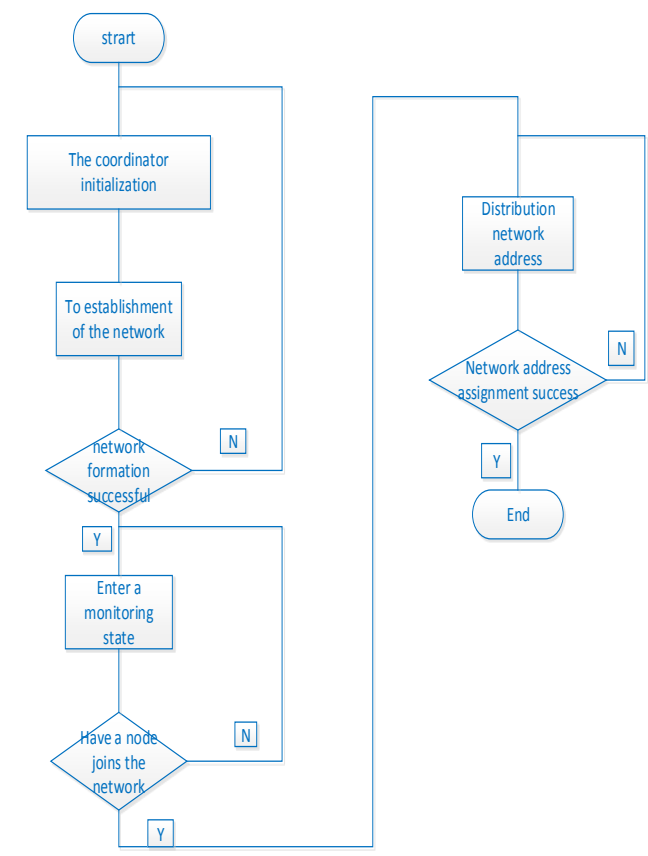

Figure 6. The ZigBee coordinator node program flowchar

\section{2) ZigBee communication module}

The working principle of software in GPRS module is as follows. Firstly, MCU gives commands to order SIM900 to get start and the DCD pin will achieve the analog-digital conversion. When the module loses connection, a high level will occur in DCD pin. Once the level is detected by DCD pin, the module will take corresponding actions to reconnect the line. When the RI pin is on the module, it will keep high level. If there come phone calls and messages, the low level will occur in RI pin and when the low level is detected by RI pin, the module will again take corresponding actions to enter into messages or phone mode. Among them, information or data about messages and phone calls will be sent to cell phone or upper computer to achieve sort management by series orders from AT+ making use of GSM network. Moreover, the systematic energy consumption will be largely reduced through the principle that the internal sleep mode will automatically turn into power down state when it is off work.

\section{3) Monitoring and management module}

Labview can use graphical author language $\mathrm{G}$ to write program and make visualized operation interface. The displaying feature of internal data collection is in the position to achieve returned value of data and visualized management with convenience.

\section{CONCLUSIONS}

Aimed at the deficiency in the existed method of detecting the thickness of icing in electric transmission line, the author optimizes the current plan and puts forward a new online detecting plan based on the Internet of things and image processing techniques.

This plan will set up a monitoring network by ZigBee, gather the collected images by DSP and recollect the information by GPRS to achieve the meshing monitor of possible icing route. Besides, the efficiency of icing detection can be enhanced and the cost of construction and maintaining can be reduced by the optimization of algorithm and the old plan.

The experiment verifies that the system is able to calculate the thickness of icing directly and through the systematic communication network to achieve the storage and alarm of icing information. This plan of monitoring system is of great feasibility, broad in detecting extent, and saving in cost; which guarantees the wide prospective of application.

\section{ACKNOWLEDGEMENT}

IN THIS PAPER, THE RESEARCH WAS SUPPORTED BY NATURE SCIENCE AND TECHNOLOGY MAJOR PROJECT OF CHINA(2011ZX4002-21).

\section{REFERENCES}

[1] SUN cai-xin, SIMA wen-xia, Su li-chun. The atmospheric environment and external electrical insulation[M]. Beijing: China Electric Power Press, 2002.

[2] WANG Zhuan-zhong, HU Xiao-guang, GENG Xin. Method of monitoring icing for transmission line based on image processing[J], CHINA MEASUREMENT \& TESTING 2011,37（2）:65

[3] MALLAT S,ZHONG S.Characterization of Signals from Multi-scale Edges[J]. IEEE Transform PAMI,1992,14(7):98-101

[4] WANG xiao-peng, Study on Monitoring the Icing-thickness of 
Transmission Line On-line Through the Imaging Method[D]. Chongqing; Chongqing University, 2009

[5] JANG xin-liang, YIhui. Icing and protection of transmission line[M].Beijing: China Electric Power Press, 2002

[6] GU Ming,MA Wenyong, QUAN Yong, HUANG Peng. Aerodynamic Force Characteristics and stabilities of Two Typical Iced Conductors[J]. Journal of Tongji University(Natural Science), 2009 (37): 1328-1332 [7] M.Farzaneh,Atmospheric Icing of Power Network[M]. New York:Springer,2008.
[8]Z.peter: Modeling and simulation of the Ice-melting Process on a Cur rent-Carrying Conductor[D]. Quebee University,2006.

[9] HU yi, Analysis and Countermeasures Discussion for Large Area Ic ing Accident onPower Grid[J].High Voltage Engineering, 2008, 34

(2) : 215-219

[10] Qin Ling-ling,Lu Lin yu.Transactions of the Chinese Society for A gricultural Machinery[J].Transactions of the Chinese Society for Agricul tural Machinery.2015,(02). 